\title{
Is a chest pain observation unit likely to be cost saving in a British hospital?
}

\author{
Steve Goodacre, Francis Morris, Jane Arnold, Karen Angelini
}

\begin{abstract}
Background-Studies from the United States (US) suggest that using a chest pain observation unit (CPOU) saves from \$567 to $\$ 2030$ per case compared with hospital admission. These savings will only be reproduced in the United Kingdom (UK) if the cost of routine hospital admission is similar. This study aimed to review current practice to determine the proportion of patients suitable for CPOU evaluation, the cost per case of routine admission and compare this with control groups in US studies.

Methods-300 patients were randomly selected from those admitted with chest pain between January and June 1998. Two independent observers reviewed the case notes to determine who would have been suitable for CPOU management. Resource use of those selected was then determined.

Results-Notes were retrieved for 285 patients. A total of $106(37.2 \%)$ were suitable for CPOU care. Mean length of stay was 51 hours (median 24). Only two patients were admitted to the coronary care unit. Interventional cardiology was limited to two angiograms, one angioplasty and one bypass graft. Estimated mean cost per patient was $£ 458$ (\$733) with interventional cardiology included, $£ 356$ (\$570) without.

Conclusion-Potential exists for the introduction of CPOU care to reduce health service costs in the UK. However, the magnitude of cost savings demonstrated in US studies were achieved by comparison to relatively high inpatient costs and
\end{abstract}

Accepted 22 March 2000 should not be extrapolated. Economic evaluation of the CPOU should be repeated in the UK. The inclusion of interventional cardiology costs is an important determinant of cost effectiveness. (Emerg Med f 2001;18:11-14)

Keywords: chest pain; cost analysis; myocardial infarction; hospital admission

At present in the UK, when a patient attends the accident and emergency (A\&E) department with chest pain, initial assessment will involve history, clinical examination and electrocardiogram (ECG). If this is insufficient to rule out myocardial infarction (MI) or other serious pathology the patient will require hospital admission.

Studies from the US suggest that the emergency department based chest pain observation unit (CPOU) is a safe and cost effective alternative to hospital admission. ${ }^{1-11}$ Patients at low to intermediate risk of MI undergo a period of intensive monitoring and cardiac enzyme testing, followed by, where appropriate, provocative testing (usually exercise treadmill). If these tests are negative the patients' discharge can be expedited. Published estimates of the cost-saving associated with CPOU use are summarised in table 1 .

A recent literature review concluded that the CPOU offered a safe and practical means of assessing patients with chest pain but questioned the applicability of cost savings to the UK. ${ }^{12}$ Before US data can be extrapolated to the UK several issues must be examined. The type of patients attending with chest pain, admission and discharge practices in the $A \& E$ department, the comparative cost of services and differences in clinical practice between the two countries may act to limit the potential for or magnitude of cost-savings associated with a CPOU.

The aim of this study therefore was to examine current practice for patients admitted to a British hospital with a primary presenting complaint of chest pain, to determine if cost-savings were possible and determine the potential magnitude of any savings.

\section{Methods}

To answer this question we needed to determine the proportion of patients who would be 
suitable for evaluation on a CPOU, the resource use when admitted and compare this with control groups in studies that have assessed the cost effectiveness of a CPOU

The Northern General Hospital (NGH) $\mathrm{A} \& \mathrm{E}$ department is the only adult $\mathrm{A} \& \mathrm{E}$ department in the city of Sheffield. It sees approximately 90000 new patients per year. High risk cardiac patients are admitted to the coronary care unit under the care of the cardiologists. Lower risk patients requiring admission are admitted under the general physicians to an acute medical admissions ward.

The A\&E computer database was used to generate a list of patients attending with a primary presenting complaint of chest pain between January and June 1998. The date of attendance and disposal (admission or discharge) was recorded for each patient. Three hundred patients were selected at random from those recorded as having been admitted and their hospital notes retrieved. Patients were excluded if their case notes could not be retrieved, if no notes relevant to the admission of interest were found or if the presenting complaint was not chest pain.

Each set of notes was independently assessed by two senior A\&E doctors (FM and SG) who are experienced in the management of acute chest pain and familiar with the literature relating to CPOUs. First they decided whether each patient might have been eligible for assessment on a CPOU by applying the list of absolute exclusion criteria outlined in appendix 1 . These criteria define a population who are at low but not negligible risk of MI. There is agreement between the protocols reported in the literature $e^{2-467910}$ on the use of these criteria and their application is not controversial.

The two assessors then applied the list of relative exclusion criteria outlined in appendix 2 . The use of these criteria varies between protocols and it would be valuable to know the potential impact of their application. Some protocols exclude patients with known ischaemic heart disease ${ }^{35}$ while others do not ${ }^{2}$ and the importance of routine exercise testing has been questioned. ${ }^{13}$ Chest pain was defined as low risk if it was either a single episode, less than one hour in duration or, if more prolonged, clearly non-cardiac in nature (sharp, stabbing, pleuritic, positional or reproduced by palpation). These definitions are derived from validated clinical predictors ${ }^{14} 15$ and have been used by some of the published CPOU protocols. ${ }^{367}$

For each case excluded, the assessors recorded which criterion/criteria applied. All disagreements were reviewed and discussed until agreement was achieved.

The notes were then searched for resource data relating to the original admission (length of stay, procedures and investigations other than routine blood tests) and follow up over the subsequent 12 months (outpatient reviews, procedures and investigations). These data were not collected for cases excluded by the absolute criteria in appendix 1 . As there is no prospect of such cases being eligible for assess- ment on a CPOU there is little to be gained from determining their resource use.

The mean cost per patient of inpatient hospital care was calculated from the length of stay using a standard rate of $£ 150.00$ per 24 hours. This defines a very broad, general cost of inpatient care, including all overheads, nursing care, professional fees, ancillary studies and pharmacy charges. As such, it reflects the cost of a basic hospital stay with reasonable accuracy but not management incorporating expensive additional procedures or investigations. We therefore added to this the average cost per patient of additional inpatient and outpatient investigations and clinic appointments, excluding interventional cardiology (angiography, percutaneous transluminal coronary angioplasty (PTCA) or coronary artery bypass grafting (CABG)). National Health Service costing methods do not easily allow separation of the costs of these procedures from that of a whole service so private practice charges were used for these costs. The total number of each procedure was multiplied by its cost and then divided by the total number of patients to give the mean cost per patient. Finally, the mean cost per patient was calculated when costs of interventional cardiology were included.

\section{Results}

Between 1 January and 30 June 19981472 patients attended the NGH A\&E department with chest pain. Some 533 (36\%) were discharged directly from A\&E while 939 (64\%) were admitted.

Of the 300 admitted patients who were selected, relevant notes were retrieved for 285 $(95 \%)$. Seventy nine $(27.7 \%)$ had no contraindication to CPOU management, while 27 $(9.5 \%)$ had a relative contraindication only (12 were unable to perform a stress test, 15 had known ischaemic heart disease), giving a total of $106(37.2 \%, 95 \%$ confidence intervals $34.3 \%$ to $40.1 \%$ ) potentially eligible for a CPOU.

Of the 179 patients excluded, cases were excluded for the following reasons: suspected or proven alternative diagnosis requiring admission, 41 cases $(14.3 \%)$; comorbidity requiring admission, 16 cases (5.6\%); ischaemic ECG, 28 cases (9.8\%); known ischaemic heart disease with high risk episode of pain, 78 cases $(27.4 \%)$; and any combination of reasons, 16 cases $(5.6 \%)$.

The potentially eligible patients had a mean age of 56 years and $57 \%$ were male. Eight patients $(7.5 \%)$ had a final diagnosis of $\mathrm{MI}$ while a further $21(19.8 \%)$ had a final diagnosis of angina. The length of stay ranged from 4 hours to 16 days. Mean length of stay was 51 hours (median 24, interquartile range 18 to 56). Only two patients were admitted to the coronary care unit (length of stay 24 and 48 hours). The investigations and procedures performed are listed in table 2. Sixty seven patients received outpatient follow up. The mean number of outpatient reviews for all patients was 1.1. 
Table 2 Investigations and procedures received by the 106 patients potentially eligible for CPOU care

\begin{tabular}{lccl}
\hline & $\begin{array}{l}\text { Total number } \\
\text { performed } \\
\text { inpatient }\end{array}$ & $\begin{array}{l}\text { Total number } \\
\text { performed as } \\
\text { outpatient }\end{array}$ & $\begin{array}{l}\text { Cost per } \\
\text { investigation/ } \\
\text { procedure }\end{array}$ \\
\hline Radiography & 111 & 1 & $£ 6.18$ \\
ECHO & 1 & 3 & $£ 75.00$ \\
Radionuclide imaging & 0 & 2 & $£ 110.00$ \\
Stress test & 0 & 35 & $£ 50.00$ \\
Angiography & 0 & 2 & $£ 537.00$ \\
PTCA & 1 & 0 & $£ 3311.00$ \\
CABG & 0 & 1 & $£ 6489.00$ \\
Others & 5 & 14 & Variable \\
\hline
\end{tabular}

The basic cost of an inpatient hospital stay is $£ 319$ (\$510). The addition of costs for investigations and procedures, excluding interventional cardiology, gives a mean cost per patient of $£ 356$ (\$570). When costs of interventional cardiology are included this rises to $£ 458$ (\$733).

\section{Discussion}

We found that approximately $37 \%$ of patients admitted after attending our A\&E department with chest pain would have been suitable for care on a CPOU. This represents about 58 patients per month. Exclusion of all patients with known ischaemic heart disease would exclude $14 \%$ of potentially eligible patients while exclusion of those unable to perform stress testing would exclude $11 \%$ of those eligible.

Studies from the US have compared resource use associated with the CPOU with that of routine hospital admission and shown costsavings associated with the CPOU varying from $\$ 567$ to $\$ 2030$ per patient. $^{235-9} 11$ These have been achieved in comparison to a mean inpatient cost per patient of $\$ 1552$ to $\$ 5116$ (table 1). By comparison the cost of routine hospital admission and subsequent investigation at our institution is much less. It therefore cannot be assumed that the magnitude of costsavings related to CPOU in the US care can be extrapolated to the UK.

Nor can the possibility of significant costsavings be ruled out. The typical length of stay on a CPOU for those who can then be discharged is 12 hours, although new diagnostic tests may make stays of four to six hours possible. ${ }^{16}$ If the referral rate from CPOU to admission or other services is kept low, savings may still be considerable. We therefore need to consider the potential determinants of cost effectiveness.

The difference in costs between our data and the US is not attributable to any difference in mean length of stay (see table 1). Instead, it is likely to be attributable to differences in health service costs and clinical practice, particularly the use of the coronary care unit and interventional cardiology.

In any economic analysis the economic viewpoint taken is a critical factor in determining which costs are measured. Studies of CPOUs typically take an institutional viewpoint to calculate the cost of assessing, diagnosing and managing the acute episode of chest pain. We have included outpatient costs in our analysis, even though this is not always done in the US studies, because the short duration of stay and limited inpatient testing at our institution means that much of the diagnostic examination is completed as an outpatient.

Whether or not to include the costs of interventional cardiology is a controversial but yet critical factor in determining cost effectiveness. Most US studies include these costs and this may be responsible for the high cost of these comparisons. Some control groups have rates of angiography as high as $20 \%$ to $25 \% .^{6}{ }^{9}$ Despite the low rate of interventional cardiology in our cohort the inclusion of these costs seem to make a dramatic difference. It could be argued that such costs are not directly related to the initial process of ruling out MI. However, if they occur as a consequence of the diagnostic process they should be taken into account. If the diagnostic testing used in a CPOU results in more referrals being made for angiography than is the case in the UK at present, then costs associated with the CPOU will likewise escalate. Such increased expenditure can only be justified if improved outcomes can be demonstrated among those referred.

Another noticeable difference between our cohort and those reported in the US is the use of the coronary care unit. A large proportion of patients admitted to hospital with chest pain in the US are admitted to the coronary care unit. In our cohort the vast majority were admitted to the general medical wards. If a CPOU is introduced to a British hospital the intensive diagnostic testing may detect cases of MI at an earlier stage and increase the number of referrals to the coronary care unit. Again, this will result in an increased use of resources and must be justified in terms of improved patient outcome.

In this study we have only considered costs, which form only half of any cost effectiveness equation. Studies from the US have demonstrated that CPOUs effectively detect cases of $\mathrm{MI}$ and very few adverse outcomes have been recorded during follow up. ${ }^{124717}$ It is therefore assumed that the effectiveness of CPOU and routine care is equivalent. This should be demonstrated, however, particularly if we intend to adopt the CPOU in a different health care system to that in which it was developed. Our data showed that some routinely admitted patients had hospital stays as short as four to six hours. As testing of these patients is limited to ECG and creatinine kinase measurement, it is debatable whether we can assume that such assessment effectively rules out MI. Likewise, many patients had to wait several months for further diagnostic testing, which would be immediately available in the CPOU. Paradoxically, it may be that the CPOU, introduced for economic reasons in the US, might be justified in terms of improved effectiveness in the UK.

Management of acute chest pain may also vary within the UK and our data may not be generalisable elsewhere. Our rate of discharge from $A \& E$ is relatively low, reflecting a cautious approach adopted by the department. It is therefore reasonable for us to assume that most potential CPOU patients will come from those 
admitted to hospital, although limitations of data availability for those discharged means that retrospective analysis cannot confirm this assumption. If, however, an A\&E department directly discharges most patients with chest pain it is likely that a large proportion of CPOU patients would otherwise have been discharged. This further undermines the potential for a CPOU to be cost-saving. The risk of inadvertent discharge of patients with $\mathrm{MI}$ ("missed MI") seems to be related to the proportion discharged after initial assessment. ${ }^{1}$ So although a CPOU is unlikely to be cost-saving in a department with a high discharge rate, it could be justified if it reduces the number of missed MIs.

This is a preliminary cost analysis and is intended to be a pilot study, examining the applicability of US data to our health care system, rather than an attempt to evaluate the cost effectiveness of a CPOU in the UK. Numbers of patients are not very large, only one hospital is analysed, the data were collected retrospectively (potentially causing bias in the selection of appropriate cases) and the costing methods are relatively crude. However, it is adequate to raise doubts about the applicability of US data to the UK. Such doubts are unlikely to be answered by a large scale, prospective analysis using detailed costing methods. Pilot units are now being established in the UK. These should be evaluated by means of an economic analysis alongside a randomised controlled trial comparing CPOU with routine care.

Cost savings from CPOU care demonstrated in US have been achieved against relatively high inpatient costs compared with those observed in our study. Nevertheless, the potential for considerable savings in the UK still exists. Economic evaluation should be repeated in the UK, analysing both costs and outcomes of CPOU care compared with routine admission. The inclusion of cardiac catheterisation and surgery costs is controversial but an important determinant of cost effectiveness.

The authors thank Mark Ellis of NGH Finance Department for supplying the hospital financial data and Jim Wardrope and the anonymous referees for their helpful comments.

Contributors

Steve Goodacre developed the study hypothesis, designed the protocol, reviewed cases for potential CPOU eligibility, collected resource use data, analysed the data and wrote the paper. Francis Morris initiated the idea of studying CPOU feasibility, reviewed cases for potential CPOU eligibility and contributed to protocol design, data analysis and writing the paper. Jane Arnold and Karen Angelini retrieved case notes for review, collected resource use data and contributed to data analysis and writing the paper.

Funding: none.

Conflicts of interest: all the authors have been involved in setting up a chest pain observation unit at the Northern General Hospital.

\section{Appendix 1}

\section{ABSOLUTE EXCLUSION CRITERIA}

1 Suspected or proven alternative diagnosis requiring admission, for example, pneumonia, pulmonary embolus, aortic dissection
2 Comorbidity requiring admission, for example, cardiac failure, shock, heart block or arrhythmia, blood pressure-systolic $>220 \mathrm{~mm} \mathrm{Hg}$ or diastolic $>110$ $\mathrm{mm} \mathrm{Hg}$

3 Ischaemic ECG changes not known to be oldgreater than $1 \mathrm{~mm}$ ST elevation or depression in two contiguous leads, $\mathrm{T}$ wave inversion in two contiguous leads or left bundle branch block

4 Known ischaemic heart disease and high risk episode of pain

\section{Appendix 2}

RELATIVE EXCLUSION CRITERIA

1 Unable to perform stress test

2 Known ischaemic heart disease and low risk episode of pain

1 Graff LG, Dallara J, Ross MA, et al. Impact on the care of the emergency department chest pain patient from the chest pain evaluation registry (CHEPER) study. Am f Cardiol 1997;80:563-8.

2 Mikhail MG, Smith FA, Gray M, et al. Cost-effectiveness of mandatory stress testing in chest pain center patients. Ann Emerg Med 1997;29:88-98.

3 Roberts RR, Zalenski RJ, Mensah EK, et al. Costs of an emergency department-based accelerated diagnostic protocol vs hospitalization in patients with chest pain. A randomized controlled trial. $\mathcal{F} A M A$ 1997;278:1670-6.

4 Gibler WB, Runyon JP, Levy RC, et al. A rapid diagnostic and treatment center for patients with chest pain in the emergency department. Ann Emerg Med 1995;25:1-8.

5 Kerns JR, Shaub TF, Fontanarosa PB. Emergency cardiac stress testing in the evaluation of emergency department patients with atypical chest pain. Ann Emerg Med 1993;22: $794-8$.

6 Gomez MA, Anderson JL, Karagounis LA, et al. An emergency department-based protocol for rapidly ruling out myocardial ischaemia reduces hospital time and expense: results of a randomized study (ROMIO). $\mathcal{f} \mathrm{Am}$ Coll Cardiol 1996;28:25-33.

7 Gaspoz J, Lee TH, Weinstein MC, et al. Cost-effectiveness of a new short-stay unit to "rule out" acute myocardial infarction in low risk patients. $\mathcal{F}$ Am Coll Cardiol 1994;24: 1249-59.

8 Rodriguez S, Cowfer JP, Lyston DJ, et al. Clinical efficacy and cost-effectiveness of rapid emergency department rule out myocardial infarction and noninvasive cardiac evaluation in patients with acute chest pain. $7 \mathrm{Am}$ Coll Cardiol 1994;23 (suppl):284A.

9 Hoekstra JW, Gibler WB, Levy RC, et al. Emergencydepartment diagnosis of acute myocardial infarction and ischaemia: a cost analysis of two diagnostic protocols. Acad Emerg Med 1994;1:103-10.

10 Farkouh ME, Smars PA, Reeder GS, et al. A clinical trial of a chest pain observation unit for patients with unstable angina. N Engl f Med 1998;339:1882-8.

11 Sayre MR, Bender AL, Dey CC, et al. Evaluating chest pain patients in an emergency department rapid diagnostic and treatment center is cost-effective. Acad Emerg Med 1994;1: A45.

12 Goodacre S. Should we establish chest pain observation units in the United Kingdom? A systematic review and critical appraisal of the literature. F Accid Emerg Med 2000; 17:1-6.

13 Lindsay J, Bonnet YD, Pinnow EE. Routine stress testing for triage of patients with chest pain: is it worth the candle? Ann Emerg Med 1998;32:600-3.

14 Goldman L, Cook EF, Brand DA, et al. A computer protocol to predict myocardial infarction in emergency department patients with chest pain. N Engl F Med 1988;318:797803.

15 Panju AA, Hemmelgarn BR, Guyatt GH, et al. The rational clinical examination. Is this patient having a myocardial infarction? $f A M A 1998 ; 280: 1256-63$.

16 Hamm CW, Goldman BW, Heeschen C, et al. Emergency room triage of patients with acute chest pain by means of rapid testing for cardiac troponin T or troponin I. N Engl F Med 1997;337:1648-53.

17 Stomel R, Grant R, Eagle KA. Lessons learned from a community hospital chest pain center. Am f Cardiol 1999;83: 1033-7. 